\title{
Investigating the differences in Web browsing behaviour of Chinese and European users using mouse tracking
}

\author{
Lee Griffiths ${ }^{1}$, Zhongming Chen ${ }^{1}$ \\ ${ }^{1}$ The School of Computing, Science and Engineering, University of Salford \\ l.s.griffiths@salford.ac.uk, z.m.chen@pgt.salford.ac.uk
}

\begin{abstract}
The World Wide Web has become a ubiquitous information source and communication channel. With such an extensive user population, it is imperative to understand how users view Web pages. Studies of Web browsing behaviour aimed at different cultures have previously been carried out using methodologies such as questionnaires, observation and expensive eye-tracking. Mouse-tracking however, has not been previously widely applied to studies of Web browsing behaviour. This paper presents an exploratory study in which Web browsing behaviour was investigated with a help of a remote proxy mouse tracker. Furthermore, this paper compares the browsing behaviour of European users with Chinese users. This comparative study tries to explore whether or not there exists any differences in expected menu positions between Chinese and European users using mouse tracking methodology.
\end{abstract}

KEYWORDS: Cross-culture, Eye-mouse Correlation, Mouse Track Patterns.

\section{Introduction}

Do users in different countries, with different ethnic origins browse Web pages based on different behaviour patterns? With the shifting geography of the global Internet population towards Asia and the Far East, Web developers need to understand whether there should be different approaches to Web application design, based on differences in the audience. Chau et al [1] suggest that the online behaviours of consumers are subtlety different in nature from traditional consumer behaviour due to the unique characteristics and interplay of technology and culture. More recently Microsoft ${ }^{\circledR}$ has recognised the need to address localisation in their Visual Studio 2005 suite allowing developers to easily automate culture mapping of Web applications. The Hong Kong and Shanghai Banking Corporation Limited (HSBC) has also long recognised the power of localisation in its marketing and business campaigns. There is no doubt the these organisations are gearing up to an anticipated geographic shift in the worlds economy.

A number of studies have in the past been carried out using a series of questionnaires or specified tasks, to explore differences in browsing behaviour in terms of user perception and satisfaction levels. Through an experiment using a series of questionnaires, Simon [2] indicates that perception and satisfaction differences exist between the cultural clusters and gender groups within Asia, Europe, Latin \& South America and North America. From a study by Han [3] the effects of alphanumerical display formatting on search time among Chinese and American users were measured with result indicating that the formatting of a list on the screen affected American users significantly with a vertical display format leading to faster responses to tasks. The formatting of a list on the screen had no significant effect on the response time among Chinese users. These and other investigations are hinting towards a need to investigate cultural differences further. 
Mouse tracking is certainly a poor man's version of eye-tracking, but it is perhaps more efficacious in that it can be easily incorporated into any Web application to provide valuable user interface usage data. Based on the evidence provided by previous studies $[4,5,6]$ of eyemouse movement correlation, it should be effective to evaluate user perception using a mouse tracking methodology. The work presented here attempted to measure the difference in search performance between Chinese and European user with regards to location of Website menus. Based on the work by McCarthy et al [7], a prediction was made that the participants of the two groups would have the same search performance times with a left-justified menu bar followed closely by the menu bar located at the top and right of the page, and finally by the bottom-justified menu bar. In addition, this study derived three mouse movement patterns after careful analysis of 320 mouse track samples.

\section{Experiment Design}

\subsection{Mouse Tracker}

The experiment was designed to collect mouse information by a proxy-based mouse tracker that could be accessed through any Web browser without altering user's browsing habits and experience. This was to ensure that the automated collection of mouse trace data was neither perceptible by the user nor intrusive. Furthermore, to prevent any delays due to network traffic, experiment pages were only displayed after all data associated with their traffic had been loaded. The use of a proxy-based mouse tracker allowed the authors to carry out the experiment without having to install the developed mouse tracking software on the experimental machines. The authors do recognise that this approach could allow test subjects to perhaps carry out the experimental tasks in an arbitrary manner however the tasks were designed to attempt to reduce any effect caused by this mode of behaviour.

\subsection{Experiment Pages}

All pages of the experiment belong to complex category [7] that is they consisted of various components such as a search box, images, email alert function and informative content, etc. All pages have a similar layout [7] which comprises of five generally accepted Web layout regions as shown in Fig. 1 below:

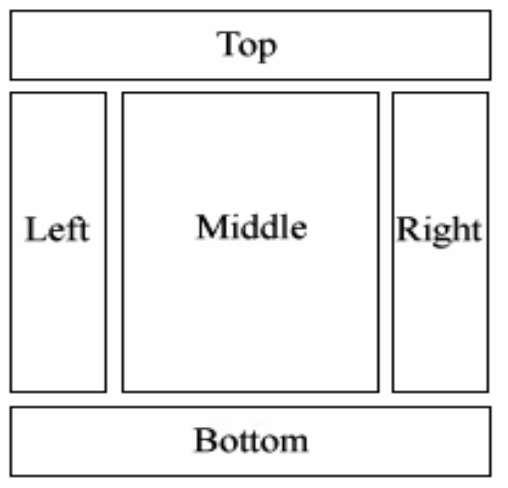

Fig. 1: This shows the layout of all pages in experiment 
All participants $(N=40)$ were tested on this kind of page layout where the menu bars were located at the top, left, right and bottom side of the experiment page. In order to examine the overall effect of different menu bars on search performance, the study was conducted using four categories of Websites ${ }^{1}$ which belong to different fields of interest respectively. Each of these pages were translated into two language versions providing for the two groups, but the layout remained the same. In addition, careful consideration was given to page design in order to avoid the impact generated by cultural knowledge. For example content relating to brands only experienced locally was either avoided of adjusted to be relevant to each cultural group. Moreover, the pages were designed with randomly allocated left, top, right and bottom menus in order to avoid the impact of attention focus and information density [8,9].

\subsection{Tasks}

It was predicted that the user perception would be one of the main factors which affected mouse trace patterns. The study also assumed that user perception could be classified into different ranks using various difficulty levels of a search task. In order to distinguish difficulty levels, each page was designed with two tasks whose relative difficulty was defined by the easy of location of the search goal. A difficult task (\#2) had only one valid goal on a page whilst an easy task (\#1) had four. It was assumed that multiple goal targets (\#1) would improve search performance and promote simple fixed pattern mouse traces (see section 5.1). Tasks goals and their locations are shown in Table 1.

Table 1: This describes the details of tasks for each experiment page.

\begin{tabular}{|c|c|c|c|}
\hline Page & Level & Scenario Description & Locations \\
\hline \multirow[t]{2}{*}{$\begin{array}{l}\text { Online } \\
\text { Movie }\end{array}$} & $\# 1$ & Please try to find the SERIES portal on the test page. & $\begin{array}{l}\text { Left, Top, } \\
\text { Right,Bottom }\end{array}$ \\
\hline & $\# 2$ & $\begin{array}{l}\text { Sign up to an EMAIL ALERT SERVICE for the latest on } \\
\text { HBO's critically-acclaimed drama. }\end{array}$ & Right \\
\hline \multirow[t]{2}{*}{ Government } & $\# 1$ & $\begin{array}{l}\text { Imagine that you are now a citizen in Cardiff. In order to } \\
\text { seek a suitable job for yourself, try to find the JOBS } \\
\text { portal. }\end{array}$ & $\begin{array}{l}\text { Left, Top, } \\
\text { Right, } \\
\text { Bottom }\end{array}$ \\
\hline & \#2 & $\begin{array}{l}\text { As a citizen in Cardiff, it is necessary to know the } \\
\text { relevant detail of the tax system. Please find and click the } \\
\text { portal for COUNCIL TAX. }\end{array}$ & Left \\
\hline \multirow[t]{2}{*}{ Education } & \#1 & $\begin{array}{l}\text { Now you are a student in Indiana University (IU), please } \\
\text { find the NEWS \& INFORMATION portal on the } \\
\text { Webpage. }\end{array}$ & $\begin{array}{l}\text { Left, Top, } \\
\text { Right, } \\
\text { Bottom }\end{array}$ \\
\hline & \#2 & $\begin{array}{l}\text { This is the second time you are going to view the } \\
\text { homepage of IU. As a new student, it would be a good } \\
\text { choice to learn the history of your own university, please } \\
\text { try to find the right portal for IU HISTORY. }\end{array}$ & Top \\
\hline \multirow[t]{2}{*}{$\begin{array}{l}\text { Online } \\
\text { Shopping }\end{array}$} & $\# 1$ & $\begin{array}{l}\text { Today, you are going to buy a cheap cooking implement } \\
\text { which is displayed in the item clearance section, so for } \\
\text { this task, try to find the CLEARANCE portal. }\end{array}$ & $\begin{array}{l}\text { Left, Top, } \\
\text { Right, } \\
\text { Bottom }\end{array}$ \\
\hline & \#2 & $\begin{array}{l}\text { After you have bought the cooking implement, } \\
\text { unfortunately it is not as good as you expected, now you } \\
\text { want to return it. For this task, please try to find the portal } \\
\text { for the RETURNSPOLICY. }\end{array}$ & Bottom \\
\hline
\end{tabular}

\footnotetext{
${ }^{1}$ Online movie site, government site, education site and online shopping site
} 
The first task of each test page involved four valid portals or targets that were allocated somewhere on the four menu bars (left, top, right and bottom) with similar information density; for the second task, there was only one valid portal which was placed on a specific menu bar and was potentially more difficult to locate.

\section{Participants}

Twenty users for each group were invited to participate in this experiment. In describing their daily Internet usages, most of participants had used the Internet for more than 2 hours; only 9 European participants used the Internet from 0-2 hours. For the European group, 20 participants consisted of 7 females and 13 males, two of them were over the age of 25 while the remaining volunteers were between 18 to 25 years old. For the Chinese group, 11 males and 9 females, only three of them were over 25 years old.

\section{Procedure}

In order to ensure high-quality data, all participants were only allowed to visit the specified experiment Web site under controlled conditions. Before the formal experiment, users were asked to complete an online background questionnaire to collect relevant demographic information. Once participants entered into the formal experiment, they carried out a total of eight search tasks following the instruction on a preceding task description page (see Table 1). Participants were only able to move to the next experiment task page after completing the preceding task by finding and clicking on the valid portal links. This process continued until all the tasks were completed. The participants were provided with a free viewing condition, in that they were told to view the pages as normal, with the opportunity to scroll up and down the page at their leisure although the authors were observant that test users did not stray from the tasks.

\section{Results}

\subsection{Mouse Movement Patterns}

Through analysing a total of 320 samples of mouse traces based on $N=40$ subjects three significant types of movement patterns were found for both groups of Chinese and European users. The following sections show these three kinds of patterns and include the relevant behavioural characteristics:

\section{Straight Pattern}

In this pattern, the mouse movement started with a pause in an initial region, while users were presumably visually browsing other regions of the page. It is surmised that once users spotted the link that they were asked to find, they moved the mouse straight to it, and usually terminated the action with a click (as shown in Fig 2.). Perhaps more importantly, the straight pattern could be further described by measuring the time taken to create the pattern. This is because some users start to move their mouse after an initial pause whilst some do not. There are completely different values for these two situations, pause and no pause, and it was assumed that there was an eye-mouse relationship if the initial suspension time of a straight pattern was below 1 or 2 seconds. Thus if there was an eye-mouse relationship then the user expectation of menu positions could be determined as well. 


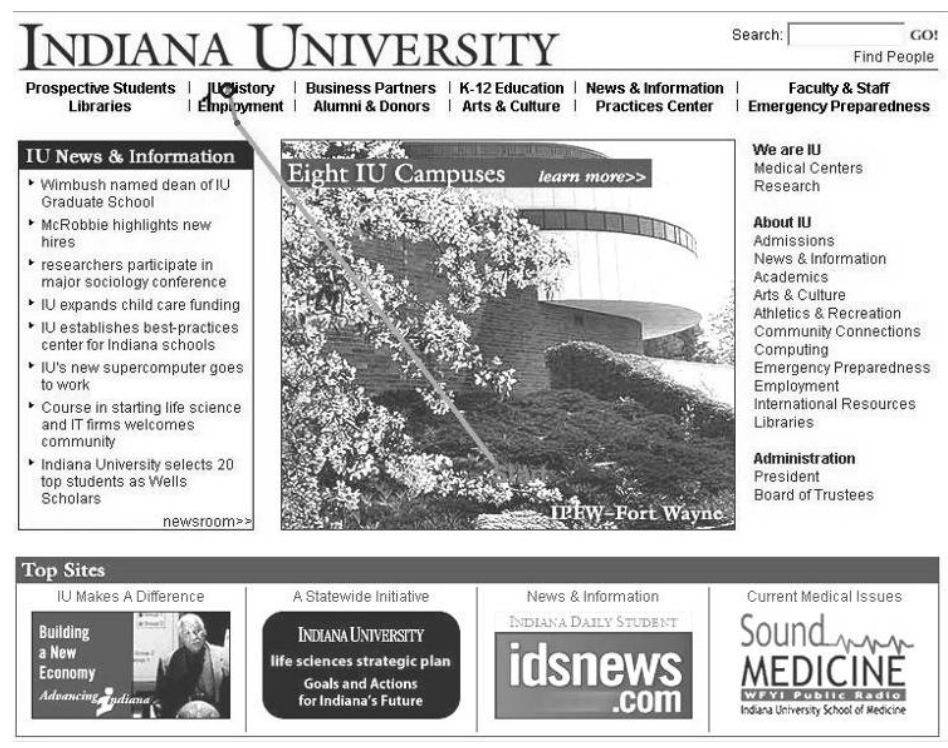

Fig. 2: Screenshot of straight pattern.

\section{Fixed Pattern}

For most users, it seems that there are some fixed regions for the mouse to stay due to their own style of operating the mouse. In this study, it was found that most users like to keep the mouse on the right-side of the page. This might be explained by the fact that they are used to moving the mouse in this area for the purposes of scrolling or unintentional clicking, whilst, they are looking at other regions. Once the destination link is found, then a straight pattern movement will be followed. Therefore it is difficult to measure user expectations of menu positions using this pattern due to the apparent random movement of the mouse and perhaps lack of relationship between the eye and mouse. However, this pattern is perhaps able to suggest hot regions for the mouse cursor to stay which could be exploited by Web designers.

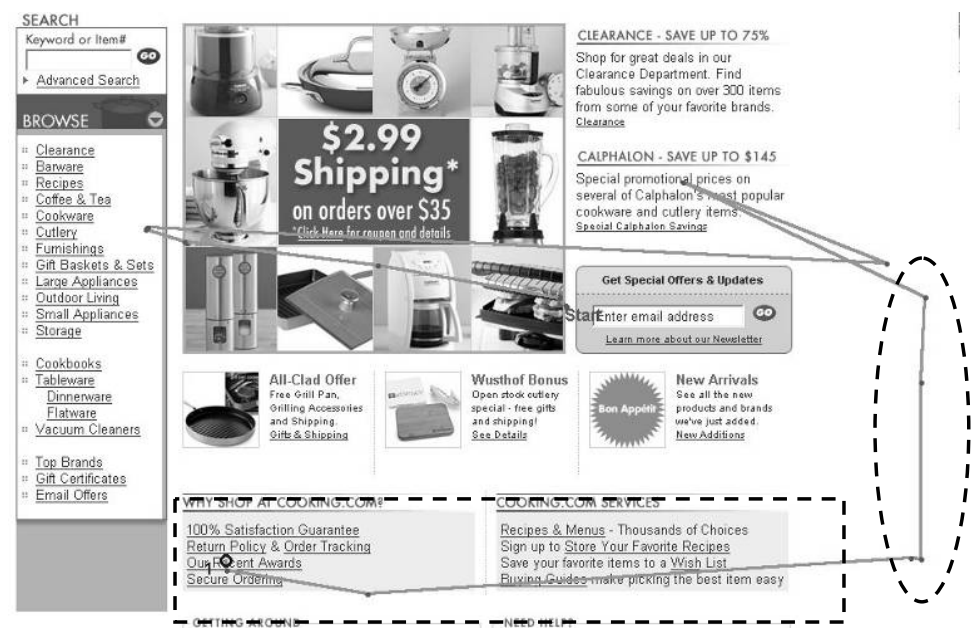

Fig. 3: Screenshot of fixed pattern.

The dotted ellipse in Fig 3 above shows, for a particular subject, that this user moves the mouse to the right-hand blank region of the page, and is doing some scrolling behaviour (up 
and down). It is assumed that they are also searching around the page at the same time as the time measurements for this trace would suggest. Ultimately, the given search task is completed with a straight pattern movement which is shown within the dotted rectangle.

\section{Guide Pattern}

In this, the most intriguing pattern, users appear to search around instinctively using the mouse cursor as some kind of guide. The data for this kind of pattern reveals a continuous movement of the mouse cursor rather than the interrupted Straight and Fixed patterns above. It is difficult to quantify this trace pattern but visually the data would suggest a relationship between mouse and eye movement, although the authors intend to investigate this further.

The trace reproduced in Fig. 4 shows a typical guide pattern sample where it can be seen that the user was searching from the left-side menu to top-side menu using a mouse cursor as a guide shown in the dotted rectangles. Subsequently, the mouse cursor was moved to the rightside blank area following a relatively fixed pattern trace and finally the task was completed with the straight pattern.

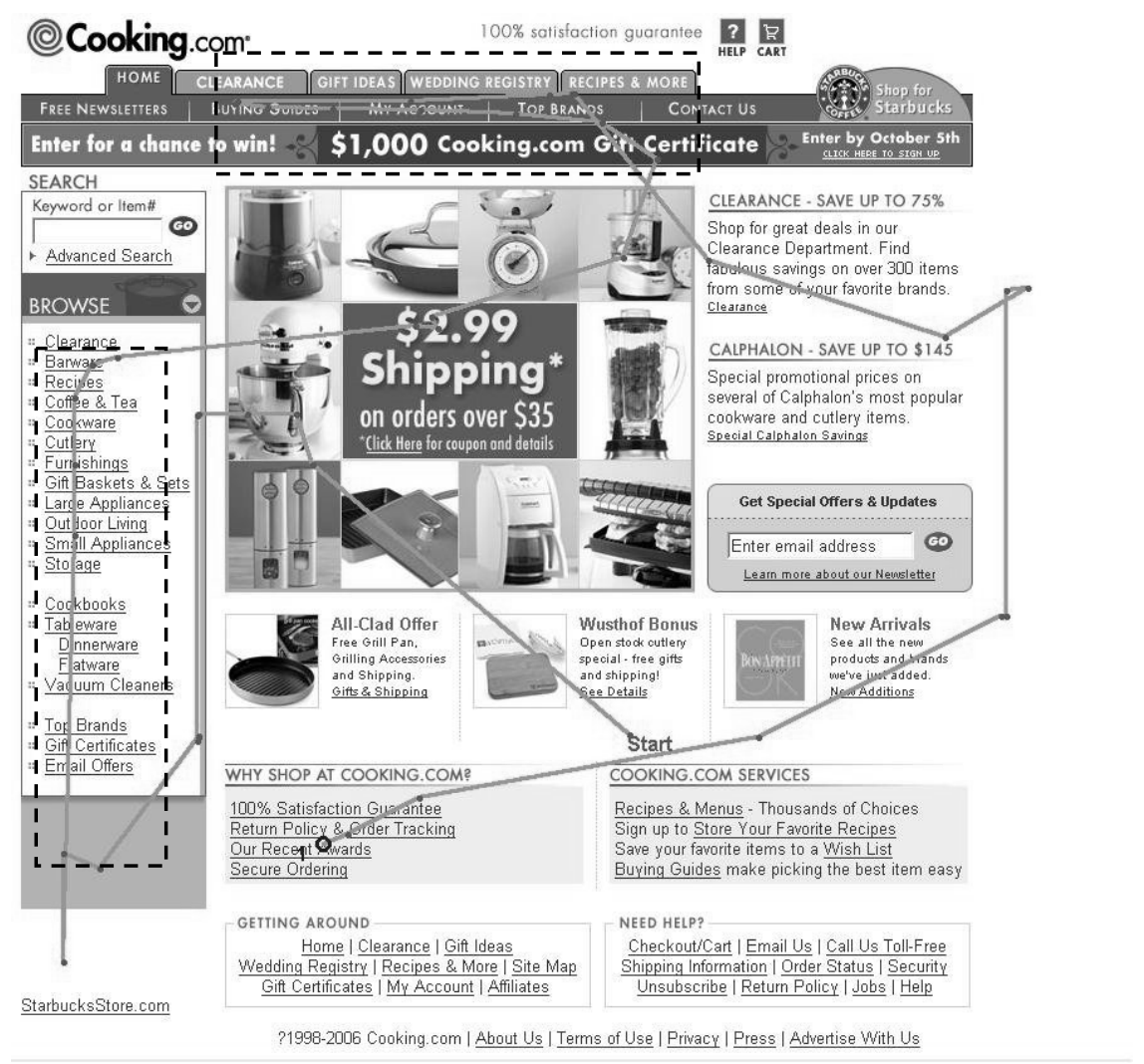

Fig. 4: The red rectangles shows a typical guide pattern trace through a menu

In contrast to the fixed pattern, the guide pattern contains very interesting information, which usability researchers can use to examine user expectations towards the position of content, be it menus or other important items. This statement does rely on the acceptance of a correlation between eye and mouse movement.

Fig. 5 below shows the proportion of the guide patterns recorded for the whole experiment (including all eight tasks). Through analysing a total 160 mouse trace samples for Chinese 
group, $44.38 \%$ of samples that contain one or more mouse tracks can be validated to be a guide pattern distinctly; and approximately 35\% for the 160 European samples. Furthermore, for all 40 subjects, there were only 5 European participants who carried out all tasks without any evidence of a guide pattern at all.

Chinese Group

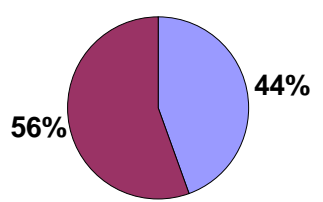

$\square$ Guide Pattern

$\square$ Other Patterns
European Group

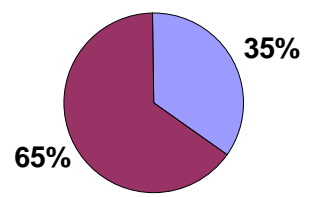

$\square$ Guide Pattern

$\square$ Other Patterns

Fig. 5: The proportion of the guide pattern in the whole experiment for both groups

Furthermore, this paper identifies a factor which appears to impact on this kind of variation on guide pattern. Fig. 6 indicates that there exists a variation due to task difficulty level. For example, a difficult to locate target is one where the search goal was in a position which did not meet the user's initial expectation. The effect is apparent for both groups of users and is displayed more frequently with the higher difficulty. Thus there could be a significant correlation between users producing a guide pattern and the location of important objects on the page.

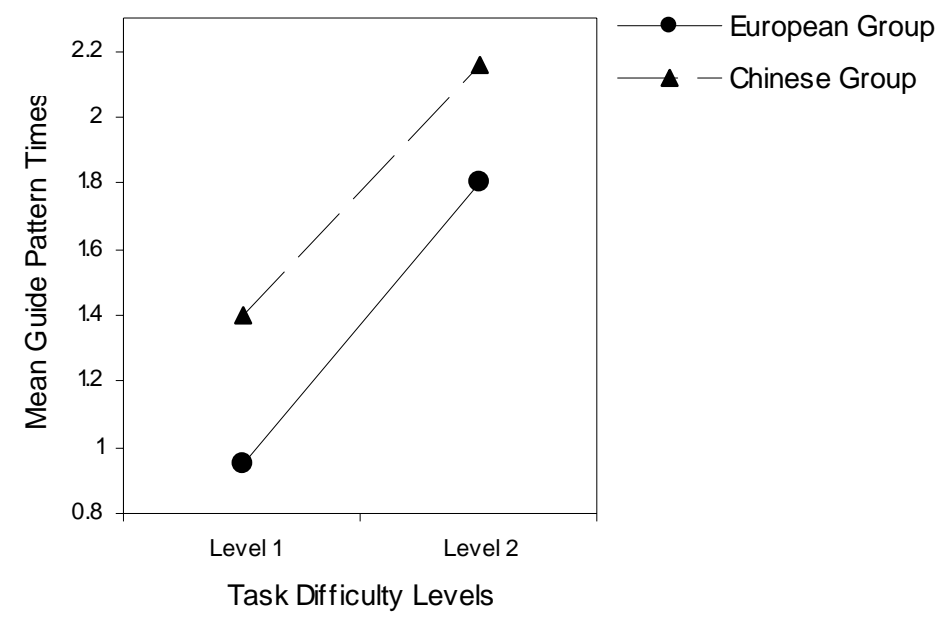

Fig. 6: The variation of proportion of guide pattern.

\subsection{Differences in Search Performance}

In order to examine differences between search performance times, ANOVA was conducted on the time measurements between two groups. The time data collected from the second tasks of each page were analysed and a summary of result are presented in Table 2. 
Table 2: ANOVA results on search performance

\begin{tabular}{|l|l|l|l|l|l|l|}
\hline ANOVA & & & & & & \\
\hline $\begin{array}{l}\text { Source of } \\
\text { Variation }\end{array}$ & SS & df & MS & F & P-value & F crit \\
\hline Sample & 84.1 & 1 & 84.1 & 1.110106 & 0.293731 & 3.903367 \\
\hline Columns & 2344.85 & 3 & 781.6167 & 10.31721 & $3.2 \mathrm{E}-06$ & 2.664109 \\
\hline Interaction & 123.35 & 3 & 41.11667 & 0.542733 & 0.653769 & 2.664109 \\
\hline Within & 11515.3 & 152 & 75.75855 & & & \\
\hline & & & & & & \\
\hline Total & 14067.6 & 159 & & & & \\
\hline
\end{tabular}

Furthermore, table 2 shows the means for each group and each menu position, so that three important results can be concluded as below:

(i) There was no significant difference between groups ("Sample") because the calculated F value (1.110106) is less than the critical F value (3.903367), $p>0.05$.

(ii) There was a significant difference for both groups $(p=0.0000032)$ for the effect caused by menu position ("Columns") overall. This shows that the position of the menu significantly affected the search performance times and also indicates that the differences in the means are not by chance alone.

(iii) It also reveals that there is no significant interaction $(p=0.653769)$ between groups and menu position.

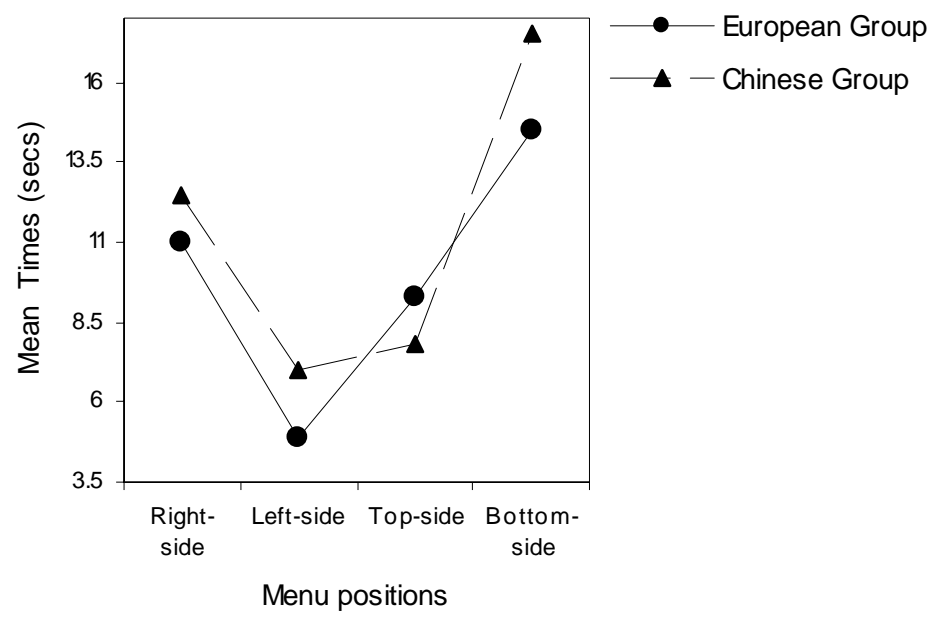

Fig. 7: Interaction effect on search performance times

Lastly from Fig. 7 above it is clear that participants of both groups spent the lowest time on left-side menu of the four. It is much more apparent for the European group than the Chinese group; however Chinese users have a similar search performance on the left-side and top-side 
menu bar around 7.5sec. As expected, both groups had the worst search performance with the bottom-side menu bar.

\section{Conclusion}

Based on a careful analysis of our data, this study has found that there are significant differences in the search performance between the different menu bar positions on both groups - indicating that the effect of changing the position of the menu bar was significant. However, it is not driven evidently depending on the region of subjects (the study has eliminated the impact of different cultures). Thus it is inferred that users in different countries would have a similar expectation of menu position.

As expected, this paper confirmed previous works that an apparent relationship exists between gaze position and cursor position on a computer screen during Web browsing, but not always.

This study thus indicates that it could be valuable to categorise mouse trace patterns in a modular way to describe the complexity of regions of a Web page.

This study thus indicates that it could be valuable to categorize mouse trace patterns in a modular way. By combining the analysis of mouse trace patterns and other variables such as time it is possible to evaluate user expectations and perceptions of page layouts.

\section{Acknowledgements}

The author would like to take this opportunity to thank all subjects who took part in the experiment.

\section{References}

1. Patrick Y. K. Chau, Melissa Cole, Anne P. Massey, Mitzi Montoya-Weiss, Robert M. O’Keefe: Cultural differences in the online behavior of consumers, ACM Press, Volume 45, (October 2002), Pages: 138 - 143

2. Steven John Simon: The impact of culture and gender on Websites: an empirical study ACM Press, Volume 32, (Winter 2001), Pages: 18 - 37

3. Songmei Han: Effects of Alphanumerical Display Formatting on Search Time among Chinese and American Users CHI '06 extended abstracts on Human factors in computing systems

4. Chen, M., Anderson, J. R., and Sohn, M.: What Can a Mouse Cursor Tell Us More? Correlation of Eye/mouse Movements on Web Browsing. Ext. Abstracts CHI 2001, ACM Press (2001).

5. Florian Mueller, Andrea Lockerd: Cheese: Tracking Mouse Movement Activity on Websites, a Tool for User Modeling

6. Ernesto Arroyo, Ted Selker, Willy Wei: Usability Tool for Analysis of Web Designs Using Mouse Tracks, CHI 2006 Work-in-Progress

7. John D. McCarthy, M. A. Sasse \& J. Riegelsberger (2003). Could I have the Menu Please? An Eyetracking Study of Design Conventions. Proceedings of HCI2003/, 8-12 Sep 2003, Bath, UK, pp. 401414.

8. Keith Rayner: Eye Movements in Reading and Information Processing: 20 Years of Research. Psychological Bulletin, 124, 3 (1998), 372-422.

9. Laura A. Granka, Helene A. Hembrooke, Geri Gay, Matthew K. Feusner: Correlates of Visual Salience and Disconnect: An Eye-tracking Evaluation 